\title{
Bing doubling and the colored Jones polynomial
}

\author{
Sakie Suzuki* \\ June 18, 2013
}

\begin{abstract}
Bing doubling is an operation which gives a satellite of a knot. It is also applied to a link by specifying a component of the link. We give a formula to compute the reduced colored Jones polynomial of a Bing double by using that of the companion. This formula enables us to compute a lot of examples of the reduced colored Jones polynomial of Bing doubles. Moreover, from this formula we can derive a divisibility property of the unified WittenReshetikhin-Turaev invariant of integral homology spheres obtained by \pm 1 -surgery along Bing doubles of knots. This result is applied to the Witten-Reshetikhin-Turaev invariant and the Ohtsuki series of these integral homology spheres.
\end{abstract}

\section{Introduction}

Bing doubling [1 is an operation which gives the satellite $B(K)$ of a framed knot $K$ as in Figure 1, i.e., $B(K)$ is the 2-component link obtained from $K$ by duplicating along its framing and making the clasps on it.

Bing doubling has been studied in the context of link concordance 2, 3, 4, 5, 7, 10, 11. Bing doubling is also important in the study of Milnor's $\bar{\mu}$ invariants [6] and finite type invariants of knots [13. Some of classical invariants such as the multivariable Alexander polynomial and the Arf invariant are useless in the study of Bing doubles since those cannot distinguish iterated Bing doubles from unlink. Cimasoni 2] used Rasmussen invariant and Cha, Livingston and Ruberman [5] used the Ozváth-Szabó invariant and the Manolescu-Owens invariant to study Bing doubles. Cochran and Melvin 8 used the quantum $S O(3)$ invariant [14 to study the Milnor degree of 3-manifolds which are obtained from $S^{3}$ by surgery along iterated Bing doubles of the Hopf link.

\footnotetext{
${ }^{*}$ Faculty of Mathematics, Kyushu University, Fukuoka, 819-0395, Japan. E-mail address: sakie@math.kyushu-u.ac.jp
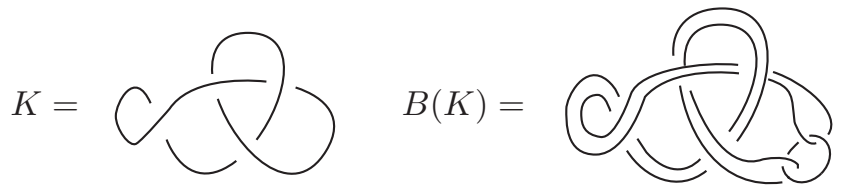

Figure 1: The trefoil knot $K$ with 4 -framing and its Bing double $B(K)$ 
Our interest here is in the relationship between Bing doubling and quantum invariants, especially the reduced colored Jones polynomial of links and the unified Witten Reshetikhin Turaev (WRT) invariant of integral homology spheres.

The colored Jones polynomial $J_{L ; W_{1}, \ldots, W_{n}} \in \mathbb{Z}\left[q^{1 / 4}, q^{-1 / 4}\right]$ is one of quantum invariants, which is defined for a framed link $L=L_{1} \cup \cdots \cup L_{n}$ with the $i$ th component colored by a finite dimensional representation $W_{i}$ of the quantized enveloping algebra $U_{h}\left(s l_{2}\right)$ of the Lie algebra $s l_{2}$.

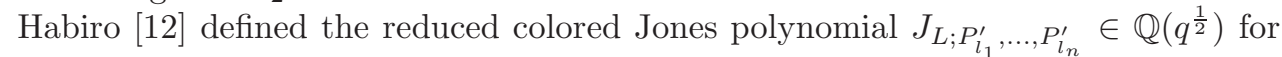
a framed link $L=L_{1} \cup \cdots \cup L_{n}$ with the $i$ th component colored by an element $P_{l_{i}}^{\prime}$ in the representation ring of $U_{h}\left(s l_{2}\right)$ over $\mathbb{Q}\left(q^{\frac{1}{2}}\right)$. By using the reduced colored Jones polynomial, he constructed the unified WRT invariant $J_{M} \in \widehat{\mathbb{Z}[q]}$ of an integral homology sphere $M$, where $\widehat{\mathbb{Z}[q]}$ is so-called Habiro ring. For each root of unity $\zeta$, we obtain the $S U(2)$ WRT invariant $\tau_{\zeta}(M) \in \mathbb{Z}[\zeta]$ from $J_{M}$ by evaluating $q=\zeta$. Here, for integral homology spheres, the $S U(2)$ WRT invariant is equal to the quantum $S O(3)$ invariant which is defined for each root of unity of odd order $\geq 3$. We can obtain also the Ohtsuki series $\tau^{O}(M) \in \mathbb{Z}[[q-1]]$ from $J_{M}$ by taking the power series expansion at $q=1$. See Sections 2.1 and 2.2 for the definitions of the reduced colored Jones polynomial and the unified WRT invariant.

In [12, Habiro proved that $J_{L ; \tilde{P}_{l_{1}}^{\prime}, \ldots, \tilde{P}_{l_{n}}^{\prime}}$ for an $n$-component, algebraically-split, 0 framed link is contained in a certain ideal $Z_{a}^{\left(l_{1}, \ldots, l_{n}\right)} \subset \mathbb{Z}\left[q, q^{-1}\right]$, where $\tilde{P}_{l}^{\prime}$ is a certain normalization of $P_{l}^{\prime}$. In [20, 21, 22, the author improved his result in the cases of Brunnian links, ribbon links, and boundary links, i.e., we proved that $J_{L ; \tilde{P}_{l_{1}}^{\prime}, \ldots, \tilde{P}_{l_{n}}^{\prime}}$ for an $n$-component, 0 -framed Brunnian link is contained in an ideal $\tilde{Z}_{B r}^{\left(l_{1}, \ldots, l_{n}\right)} \subset Z_{a}^{\left(l_{1}, \ldots, l_{n}\right)}$, and $J_{L ; \tilde{P}_{l_{1}}^{\prime}, \ldots, \tilde{P}_{l_{n}}^{\prime}}$ for an $n$-component, 0 -framed ribbon or boundary link is contained in an ideal $Z_{r, b}^{\left(l_{1}, \ldots, l_{n}\right)} \subset \tilde{Z}_{B r}^{\left(l_{1}, \ldots, l_{n}\right)}$. These results were proved by using the universal $s l_{2}$ invariant of bottom tangles. In [23], the author also proved that the ideals $\tilde{Z}_{B r}^{\left(l_{1}, \ldots, l_{n}\right)}$ and $Z_{r, b}^{\left(l_{1}, \ldots, l_{n}\right)}$ are principal, and gave the generators of these ideals. (In [22, 23], there appears another ideal $Z_{B r}^{\left(l_{1}, \ldots, l_{n}\right)} \subset \mathbb{Z}\left[q, q^{-1}\right]$ such that $\tilde{Z}_{B r}^{\left(l_{1}, \ldots, l_{n}\right)}=Z_{B r}^{\left(l_{1}, \ldots, l_{n}\right)} \cup Z_{a}^{\left(l_{1}, \ldots, l_{n}\right)}$.)

For a framed link $L=L_{1} \cup \cdots \cup L_{n}$, we denote by $B(L ; 1)$ the $(n+1)$-component link obtained from $L$ by applying Bing doubling to $L_{1}$. It is not difficult to see that the reduced colored Jones polynomial of $B(L ; 1)$ is a linear combination of those of $L$ (Lemma 3.1), i.e., we have

$$
J_{B(L ; 1) ; P_{i}^{\prime}, P_{j}^{\prime}, P_{l_{2}}^{\prime}, \ldots, P_{l_{n}}^{\prime}}=\sum_{l \geq 0} x_{i, j}^{(l)} J_{L ; P_{l}^{\prime}, P_{l_{2}}^{\prime}, \ldots, P_{l_{n}}^{\prime}}
$$

for certain $x_{i, j}^{(l)} \in \mathbb{Q}\left(q^{\frac{1}{2}}\right)$.

One of two main results in this paper is Theorem 3.2. which gives the coefficients $x_{i, j}^{(l)}$ explicitly. This result gives many examples of computations of the reduced colored Jones polynomials of Bing doubles.

The other main result is Theorem 3.4 which says a divisibility property of the difference $J_{\left.M\left(B(K) ; \epsilon, \epsilon^{\prime}\right)\right)}-J_{M}$ of unified WRT invariants, where $M$ is an integral homology sphere and $K$ is a 0 -framed knot in $M$, and $M\left(B(K) ; \epsilon, \epsilon^{\prime}\right)$ is the integral homology 
sphere obtained from $M$ by surgery along the Bing double $B(K)$ with $\epsilon, \epsilon^{\prime} \in\{ \pm 1\}$ framings. This result is applied to prove some improvements of Habiro's results in 12 . for the WRT invariant and the Ohtsuki series of integral homology spheres.

The rest of the paper is organized as follows. In Section 2 we recall the definitions of the reduced colored Jones polynomial and the unified WRT invariant. In Section 3 ,

we give the main results. In Section 4 we study the coefficients $x_{i, j}^{(l)} \in \mathbb{Q}\left(q^{\frac{1}{2}}\right)$ further. In Section 5. we compute some of the reduced colored Jones polynomials of Milnor's link as in Figure 4, which is obtained from the Borromean rings by applying Bing doubling repeatedly. Section 6 is devoted to the proofs.

\section{Preliminaries}

In this section, we recall from [12 the definition of the reduced colored Jones polynomial and the unified WRT invariant.

We use the following $q$-integer notations:

$$
\begin{aligned}
& \{i\}=q^{i / 2}-q^{-i / 2}, \quad\{i\}_{n}=\{i\}\{i-1\} \cdots\{i-n+1\}, \\
& \{n\} !=\{n\}_{n}, \quad\left[\begin{array}{c}
i \\
n
\end{array}\right]=\{i\}_{n} /\{n\} !
\end{aligned}
$$

for $i \in \mathbb{Z}, n \geq 0$.

\subsection{Reduced Colored Jones polynomial}

The colored Jones polynomial $J_{L ; W_{1}, \ldots, W_{n}} \in \mathbb{Z}\left[q^{1 / 4}, q^{-1 / 4}\right]$ is defined for an $n$-component framed link $L$ with the $i$ th component $L_{i}$ colored by a finite dimensional representation $W_{i}$ of the quantized enveloping algebra $U_{h}\left(s l_{2}\right)$ of the Lie algebra $s l_{2}$. In this paper, we follow 12 for the definition of colored Jones polynomial. The reduced colored Jones polynomial is defined as a linear combination of the colored Jones polynomial as follows.

For $m \geq 0$, let $V_{m}$ denote the $(m+1)$-dimensional irreducible representation of $U_{h}\left(s l_{2}\right)$. Let $\mathcal{R}$ denote the representation ring of $U_{h}\left(s l_{2}\right)$ over $\mathbb{Q}\left(q^{\frac{1}{2}}\right)$, i.e., $\mathcal{R}$ is the $\mathbb{Q}\left(q^{\frac{1}{2}}\right)$-algebra

$$
\mathcal{R}=\operatorname{Span}_{\mathbb{Q}\left(q^{\frac{1}{2}}\right)}\left\{V_{m} \mid m \geq 0\right\}
$$

with the multiplication induced by the tensor product.

We define the colored Jones polynomial of an $n$-component framed link $L$ with the $i$ th component $L_{i}$ colored by $X_{i}=\sum_{l_{i} \geq 1} a_{l_{i}}^{(i)} V_{l_{i}} \in \mathcal{R}$ by

$$
J_{L ; X_{1}, \ldots, X_{n}}=\sum_{l_{1}, \ldots, l_{n} \geq 1} a_{l_{1}}^{(1)} \cdots a_{l_{n}}^{(n)} J_{L ; V_{l_{1}}, \ldots, V_{l_{n}}} \in \mathbb{Q}\left(q^{\frac{1}{2}}\right) .
$$

For $l \geq 0$, set

$$
P_{l}^{\prime}=\frac{1}{\{l\} !} \prod_{i=0}^{l-1}\left(V_{1}-q^{i+\frac{1}{2}}-q^{-i-\frac{1}{2}}\right) \in \mathcal{R} .
$$


For an $n$-component framed link $L$, we call $J_{L ; P_{l_{1}}^{\prime}, \ldots, P_{l_{n}}^{\prime}}$ the reduced colored Jones polynomial of $L$.

\subsection{Unified WRT invariant}

For $k \geq 0$, set

$$
\mathcal{P}_{k}=\operatorname{Span}_{\mathbb{Z}\left[q, q^{-1}\right]}\left\{q^{-\frac{1}{4} l(l-1)} P_{l}^{\prime} \mid l \geq k\right\} \subset \mathcal{R},
$$

Set

$$
\hat{\mathcal{P}}=\lim _{k \geq 0} \mathcal{P}_{0} / \mathcal{P}_{k}
$$

Set

$$
\omega^{ \pm 1}=\sum_{l=0}^{\infty}( \pm 1)^{l} q^{ \pm \frac{1}{4} l(l+3)} P_{l}^{\prime} \in \hat{\mathcal{P}} .
$$

Let $\widehat{\mathbb{Z}[q]}$ be the Habiro ring, i.e.,

$$
\widehat{\mathbb{Z}[q]}=\lim _{n \geq 0} \mathbb{Z}[q] /\left((1-q)\left(1-q^{2}\right) \cdots\left(1-q^{n}\right)\right) .
$$

Let $M$ be the integral homology sphere obtained by surgery along an algebraicallysplit link $L=L_{1} \cup \cdots \cup L_{n}$ in $S^{3}$ with framings $\epsilon_{1}, \ldots, \epsilon_{n} \in\{ \pm 1\}$. Habiro 12] constructed the unified WRT invariant $J_{M} \in \widehat{\mathbb{Z}[q]}$ of $M$ by

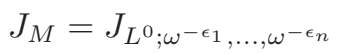

$$
\begin{aligned}
& :=\sum_{l_{1}, \ldots, l_{n}=0}^{\infty}\left(\prod_{i=1, \ldots, n}\left(-\epsilon_{i}\right)^{l_{i}} q^{-\epsilon_{i} \frac{1}{4} l_{i}\left(l_{i}+3\right)}\right) J_{L^{0} ; P_{l_{1}}^{\prime}, \ldots, P_{l_{n}}^{\prime}} \in \widehat{\mathbb{Z}[q]},
\end{aligned}
$$

where $L^{0}$ is the link obtained from $L$ by changing all framings to 0 .

For an integral homology sphere $M$ and a root of unity $\zeta$, let $\tau_{\zeta}(M) \in \mathbb{Z}[\zeta]$ be the WRT invariant [19] at $\zeta$. Let $\mathrm{ev}_{\zeta}: \widehat{\mathbb{Z}[q]} \rightarrow \mathbb{Z}[\zeta]$ be the evaluation map. For each root of unity, the WRT invariant is obtained from the unified WRT invariant as follows.

Theorem 2.1 (Habiro [12]). Let $M$ be an integral homology sphere. For each root of unity $\zeta$, we have

$$
\operatorname{ev}_{\zeta}\left(J_{M}\right)=\tau_{\zeta}(M)
$$

For an integral homology sphere $M$, let $\tau^{O}(M)$ denote the Ohtsuki series [16] and $\imath\left(J_{M}\right) \in \mathbb{Z}[[q-1]]$ denote the power series expansion of $J_{M}$ at $q=1$.

Theorem 2.2 (Habiro [12]). For an integral homology sphere $M$, we have

$$
\imath\left(J_{M}\right)=\tau^{O}(M) .
$$




\section{Main results}

In this section, we give the main results of this paper for the reduced colored Jones polynomial (Theorem 3.2) and the unified WRT invariant (Theorem 3.4). We give also applications to the WRT invariant (Proposition 3.10) and the Ohtsuki series (Proposition 3.12).

\subsection{Result for the reduced colored Jones polynomial}

It is known that the colored Jones polynomial of a satellite is a linear combination of the colored Jones polynomials of the companion [18, 15]. The following lemma says that a similar situation works for the reduced colored Jones polynomials of Bing doubles.

Lemma 3.1. There exists $x_{i, j}^{(l)} \in \mathbb{Q}\left(q^{1 / 2}\right), i, j, l \geq 0$, such that

$$
J_{B(L ; 1) ; P_{i}^{\prime}, P_{j}^{\prime}, W_{1}, \ldots, W_{n-1}}=\sum_{l \geq 0} x_{i, j}^{(l)} J_{L ; P_{l}^{\prime}, W_{1}, \ldots, W_{n-1}},
$$

for any $n$-component framed link $L=L_{1} \cup \cdots \cup L_{n}$ and $W_{1}, \ldots, W_{n-1} \in \mathcal{R}$.

Proof. For simplicity, we prove the claim for $n=1$, i.e., $L=K$ is a framed knot. We can prove the assertion for $n \geq 2$ similarly. Since $\left\{V_{l}\right\}_{l \geq 0}$ is a basis of $\mathcal{R}$, the colored Jones polynomial $J_{B(K) ; P_{i}^{\prime}, P_{j}^{\prime}}$ is a linear sum of colored Jones polynomials $J_{B(K) ; V_{t}, V_{u}}$ in $\mathcal{R}$. Since the colored Jones polynomial $J_{B(K) ; V_{t}, V_{u}}$ is a linear sum of colored Jones polynomials $J_{K ; V_{s}}$ in $\mathbb{Z}\left[q^{1 / 2}, q^{-1 / 2}\right]$ (cf. [15, Theorem 3.1]), and since $\left\{P_{l}^{\prime}\right\}_{l \geq 0}$ is also a basis of $\mathcal{R}, J_{B(K) ; V_{t}, V_{u}}$ is a linear sum of colored Jones polynomials $J_{K ; P_{l}^{\prime}}$ in $\mathcal{R}$. Consequently, $J_{B(K) ; P_{i}^{\prime}, P_{j}^{\prime}}$ is a linear sum of $J_{K ; P_{l}^{\prime}}$ in $\mathcal{R}$. Moreover, in each step, the coefficients of the linear sum do not depend on the knot $K$. Hence we have the assertion.

One of our main results is the following, which we prove in Section 6.1

Theorem 3.2. For $i, j, l \geq 0$, we have

$$
x_{i, j}^{(l)}=\delta_{i, j}(-1)^{i}\{l\} ! \alpha_{i, l},
$$

where

$$
\alpha_{i, l}=\sum_{k=0}^{i}(-1)^{k}\left[\begin{array}{c}
2 i+1 \\
k
\end{array}\right]\left[\begin{array}{c}
2 i+l-2 k+1 \\
2 l+1
\end{array}\right] .
$$

Theorem 3.2 enables us to compute many examples of the reduced colored Jones polynomial of Bing doubles. Especially in Section 5 , we give examples with Milnor's links, which are obtained from the Borromean rings by applying Bing doubling repeatedly. Moreover, from Theorem 3.2 we can derive a divisibility property of the unified WRT invariant as in the following section. 


\subsection{Result for the unified WRT invariant}

For $m \geq 1$, let $\Phi_{m}=\prod_{d \mid m}\left(q^{d}-1\right)^{\mu\left(\frac{m}{d}\right)} \in \mathbb{Z}[q]$ denote the $m$ th cyclotomic polynomial, where $\prod_{d \mid m}$ denotes the product over all the positive divisors $d$ of $m$, and $\mu$ is the Möbius function.

Let $M$ be an integral homology sphere, and $L=L_{1} \cup \cdots \cup L_{n}$ an algebraically-split link in $M$. For $\epsilon_{1}, \ldots, \epsilon_{n} \in\{ \pm 1\}$, let $M\left(L ; \epsilon_{1}, \ldots, \epsilon_{n}\right)$ denote the integral homology sphere obtained from $M$ by surgery along $L$ with framings $\epsilon_{1}, \ldots, \epsilon_{n}$.

In what follows, two integral homology spheres $M$ and $M^{\prime}$ are said to be related by a special Bing double surgery if there is a 0 -framed knot $K$ in $M$ and signs $\epsilon, \epsilon^{\prime} \in\{ \pm 1\}$ such that $M\left(B(K) ; \epsilon, \epsilon^{\prime}\right)$ is orientation-preserving homeomorphic to $M^{\prime}$.

Remark 3.3. Note that $M\left(B(K) ; \epsilon, \epsilon^{\prime}\right)=M\left(W_{\epsilon^{\prime}}(K), \epsilon\right)$, where $W_{\epsilon^{\prime}}(K)$ is the Whitehead double of $K$ with a clasp of $\epsilon^{\prime}$-type.

The other main result in this paper is the following, which we prove in Section 6.2.

Theorem 3.4. Let $M$ and $M^{\prime}$ be integral homology spheres related by a special Bing double surgery. Then we have

$$
J_{M^{\prime}}-J_{M} \in \Phi_{1}^{2} \Phi_{2}^{2} \Phi_{3} \Phi_{4} \Phi_{6} \widehat{\mathbb{Z}[q]}=\left(q^{4}-1\right)\left(q^{6}-1\right) \widehat{\mathbb{Z}[q]} .
$$

In particular, if $M=S^{3}$, then we have

$$
J_{M^{\prime}}-1 \in \Phi_{1}^{2} \Phi_{2}^{2} \Phi_{3} \Phi_{4} \Phi_{6} \widehat{\mathbb{Z}[q]} .
$$

Remark 3.5. Note that Theorem 3.4 implies (2) for integral homology spheres $M$ and $M^{\prime}$ related by a sequence of special Bing double surgeries, i.e., when there is a sequence $M=M_{1}, M_{2}, \ldots, M_{l}=M^{\prime}$ of integral homology spheres such that for each $i=1, \ldots, l-1, M_{i}$ and $M_{i+1}$ are related by a special Bing double surgery.

Remark 3.6. Habiro's result [12, Proposition 12.15] implies that for integral homology spheres $M$ and $M^{\prime}$ with the same Casson invariant, we have

$$
J_{M^{\prime}}-J_{M} \in \Phi_{1}^{2} \Phi_{2} \Phi_{3} \Phi_{4} \Phi_{6} \widehat{\mathbb{Z}[q]}=(q-1)\left(q^{2}+1\right)\left(q^{6}-1\right) \widehat{\mathbb{Z}[q]} .
$$

Since $M^{\prime}$ and $M$ in Theorem 3.4 has the same Casson invariant, Theorem 3.4 is an improvement of his result with respect to $\Phi_{2}$.

Remark 3.7. In 9, Cochran and Melvin introduced the quantum p-order $\mathfrak{o}_{p}(M)$ for a prime $p$ and a 3-manifold $M$, which is the p-order of the quantum $S O(3)$ invariant $\tau_{\zeta_{p}}^{S O(3)}(M) \in \mathbb{Q}\left(\zeta_{p}\right)$ associated with a primitive $p$ th root of unity $\zeta_{p}$. Here, the $p$ order of an element $g$ in the cyclotomic field $\mathbb{Q}\left(\zeta_{p}\right)$ is the exponent of the prime ideal $\left(\zeta_{p}-1\right) \subset \mathbb{Z}\left[\zeta_{p}\right]$ in the prime decomposition of the fractional ideal generated by $g$.

For prime $p>3$, they [8, Section 3] proved that

$$
\hat{\mathfrak{o}}_{p}\left(S^{3}\left(B(L ; 1) ; 0,0, f_{2}, \ldots, f_{n}\right)\right)=\hat{\mathfrak{o}}_{p}\left(S^{3}\left(L ; 0, f_{2}, \ldots, f_{n}\right)\right)+1
$$

for an $n$-component link $L$ and integers $f_{2}, \ldots, f_{n}$, where $\hat{\mathfrak{o}}_{p}=\mathfrak{o}_{p} \cdot \frac{2}{(p-3)}$ is a rescaling version of $\mathfrak{o}_{p}$. Though Theorem 3.4 appears to say nothing about the quantum $p$-order of $S^{3}\left(B(K) ; \epsilon, \epsilon^{\prime}\right)$, Theorem 3.2 could be applied to study the quantum $p$-order of 3manifolds obtained from $S^{3}$ by surgery along Bing doubles with arbitrary framings. 


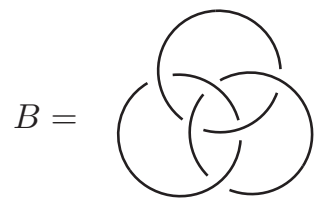

Figure 2: Borromean rings $B$

There is a family of examples of integral homology spheres which do not have the divisibility property in Theorem 3.4 as follows.

Let $B$ be the Borromean rings depicted in Figure 2 and $B_{1}, B_{2}, B_{3}$ the components of $B$. For $i, j, k \in \mathbb{Z}$, let $M_{i, j, k}$ be the integral homology sphere obtained from $S^{3}$ by surgery along $B_{1}, B_{2}, B_{3}$ with framings $-1 / i,-1 / j,-1 / k$, respectively.

We prove the following proposition in Section 6.3

Proposition 3.8. For $i, j, k \in \mathbb{Z}$, we have

$$
J_{M_{i, j, k}}-1 \equiv 6 i j k \Phi_{2} \quad\left(\bmod \Phi_{2}^{2}\right) .
$$

Note that the Casson invariant of $M_{i, j, k}$ is $-6 i j k$. It appears to be natural to raise the following conjecture.

Conjecture 3.9. For integral homology spheres $M$ and $M^{\prime}$ with the same Casson invariant, we have

$$
J_{M^{\prime}}-J_{M} \in \Phi_{1}^{2} \Phi_{2}^{2} \Phi_{3} \Phi_{4} \Phi_{6} \widehat{\mathbb{Z}[q]} .
$$

\subsection{Applications to the WRT invariant}

From Theorem 3.4 by substituting a root of unity $\zeta$ for $q$, we obtain the following result for the WRT invariant.

Proposition 3.10. Let $M$ and $M^{\prime}$ be integral homology spheres related by a special Bing double surgery. For any root of unity $\zeta$, we have

$$
\tau_{\zeta}\left(M^{\prime}\right)-\tau_{\zeta}(M) \in\left(\zeta^{4}-1\right)\left(\zeta^{6}-1\right) \mathbb{Z}[\zeta] .
$$

Proposition 3.10improves the result obtained from Habiro's result (3) by substituting $\zeta$ for $q$, in the case of $\zeta=\zeta_{2 p^{e}}$ as follows. Here, $\zeta_{2 p^{e}}$ denotes a primitive $2 p^{e}$ th root of unity for a prime $p$ and an integer $e \geq 0$.

Corollary 3.11. Let $M$ and $M^{\prime}$ be integral homology spheres related by a special Bing double surgery.

(i) For e $\geq 0$, we have

$$
\tau_{\zeta_{2} e}\left(M^{\prime}\right)-\tau_{\zeta_{2} e}(M) \in\left(\zeta_{2^{e}}^{4}-1\right)\left(\zeta_{2^{e}}^{2}-1\right) \mathbb{Z}\left[\zeta_{2^{e}}\right] .
$$

(ii) For $e \geq 0$, we have

$$
\tau_{\zeta_{2 \cdot 3^{e}}}\left(M^{\prime}\right)-\tau_{\zeta_{2 \cdot 3^{e}}}(M) \in\left(\zeta_{2 \cdot 3^{e}}+1\right)^{2}\left(\zeta_{2 \cdot 3^{e}}^{2}-\zeta_{2 \cdot 3^{e}}+1\right) \mathbb{Z}\left[\zeta_{2 \cdot 3^{e}}\right] .
$$

(iii) For a prime $p>3$ and $e \geq 0$, we have

$$
\tau_{\zeta_{2 p^{e}}}\left(M^{\prime}\right)-\tau_{\zeta_{2 p^{e}}}(M) \in\left(\zeta_{2 p^{e}}+1\right)^{2} \mathbb{Z}\left[\zeta_{2 p^{e}}\right] .
$$




\subsection{Applications to the Ohtsuki series}

Theorem 3.4 can also be applied to the Ohtsuki series as follows.

For an integral homology sphere $M$, we write

$$
\tau^{O}(M)=\imath\left(J_{M}\right)=1+\sum_{i \geq 1} \lambda_{i}(M) \hbar^{i} \in \mathbb{Z}[[\hbar]],
$$

where we set $\hbar=(q-1)$.

For integral homology spheres $M, M^{\prime}$, set

$$
\Lambda_{k}\left(M, M^{\prime}\right)=\lambda_{k}\left(M^{\prime}\right)-\lambda_{k}(M)
$$

for $k \geq 1$. Note that $\Lambda_{k}\left(S^{3}, M^{\prime}\right)=\lambda_{k}\left(M^{\prime}\right)$.

For integral homology spheres $M$ and $M^{\prime}$ with the same Casson invariant, Habiro's result [12, Proposition 12.28] implies

$$
\sum_{i=0}^{k} b_{i} \Lambda_{k-i+2}\left(M, M^{\prime}\right) \equiv 0(\bmod \mathbb{Z})
$$

for $k \geq 0$ and a certain series $b_{i} \in \mathbb{Z}\left[\frac{1}{6}\right]$ with $b_{0}=1 / 12$.

We can improve his result as follows.

Proposition 3.12. Let $M$ and $M^{\prime}$ be integral homology spheres related by a special Bing double surgery. For $k \geq 0$, we have

$$
\sum_{i=0}^{k} c_{i} \Lambda_{k-i+2}\left(M, M^{\prime}\right) \equiv 0(\bmod \mathbb{Z})
$$

where $c_{0}=1 / 24, c_{1}=-1 / 8, c_{2}=59 / 288, c_{3}=-17 / 72, \ldots \in \mathbb{Z}\left[\frac{1}{6}\right]$ are determined by

$$
\begin{aligned}
\sum_{i \geq 0} c_{i} \hbar^{i} & =\frac{1}{(q+1)^{2}\left(q^{2}+q+1\right)\left(q^{2}+1\right)} \\
& =\frac{1}{24+72 \hbar+98 \hbar^{2}+76 \hbar^{3}+35 \hbar^{4}+9 \hbar^{5}+\hbar^{6}} .
\end{aligned}
$$

In particular, for $k \geq 2, \Lambda_{k+2}\left(M, M^{\prime}\right)(\bmod 24)$ is determined by $\Lambda_{i}\left(M, M^{\prime}\right)$ for $i=$ $2, \ldots, k$.

Proof. The proof is similar to that of [12, Proposition 12.16] if we use Theorem 3.4 instead of [12, Proposition 12.14] and $\Lambda_{i}$ instead of $\lambda_{i}$. The latter part follows from $c_{0}=1 / 24$ and $c_{2}=-1 / 8 \in(1 / 24) \mathbb{Z}$.

Remark 3.13. In [24, Corollary 4.1], Takata determined the image of $\lambda_{k}, k=1, \ldots, 6$, for integral homology spheres. The congruence relations (4) for $M=S^{3}, k=1, \ldots, 6$, can be derived also from her result. Note that the relation (v) in [24, Corollary 4.1] contains an error which can be corrected by using [24, Theorem 3.1]. 


\section{Properties of the coefficients $x_{i, j}^{(l)}$ in Theorem 3.2.}

In this section, we study the coefficients $x_{i, j}^{(l)}=\delta_{i, j}(-1)^{i}\{l\} ! \alpha_{i, l}$ which appears in Theorem 3.2 .

\subsection{Symmetry property}

The element $\alpha_{m, n}$ has a symmetry property as follows, which we prove in Section 6.1

Lemma 4.1. For $m, n \geq 0$, we have

$$
\alpha_{m, n}=\frac{\{2 m+1\} !}{\{2 n+1\} !} \alpha_{n, m} .
$$

Corollary 4.2. For $m, n \geq 0$, we have

$$
x_{m, m}^{(n)}=(-1)^{m+n} \frac{\{2 m+1\} !\{n\} !}{\{2 n+1\} !\{m\} !} x_{n, n}^{(m)} .
$$

Proof. By (11) and Lemma 4.1, we have

$$
\begin{aligned}
x_{m, m}^{(n)} & =(-1)^{m}\{n\} ! \alpha_{m, n} \\
& =(-1)^{m}\{n\} ! \frac{\{2 m+1\} !}{\{2 n+1\} !} \alpha_{n, m} \\
& =(-1)^{m}\{n\} ! \frac{\{2 m+1\} !}{\{2 n+1\} !}\left((-1)^{n} \frac{1}{\{m\} !} x_{n, n}^{(m)}\right) \\
& =(-1)^{m+n} \frac{\{2 m+1\} !\{n\} !}{\{2 n+1\} !\{m\} !} x_{n, n}^{(m)} .
\end{aligned}
$$

Hence we have the assertion.

\subsection{Particular values}

For $m, n \geq 0$, note that

$$
\alpha_{m, n}=\sum_{k=0}^{\left\lfloor m-\frac{n}{2}\right\rfloor}(-1)^{k}\left[\begin{array}{c}
2 m+1 \\
k
\end{array}\right]\left[\begin{array}{c}
2 m+n-2 k+1 \\
2 n+1
\end{array}\right],
$$

where the upper bound comes from

$$
\left[\begin{array}{c}
2 m+n-2 k+1 \\
2 n+1
\end{array}\right]=0
$$

for $2 m+n-2 k+1<2 n+1$, i.e., for $k>m-n / 2$.

We can compute particular values of $\alpha_{m, n}$ as follows.

Proposition 4.3. We have 
(i) $\alpha_{m, n}=0$ unless $\frac{n}{2} \leq m \leq 2 n$,

(ii) $\alpha_{m, 2 m}=1$ for $m \geq 0$, and

(iii) $\alpha_{m, 2 m-1}=\{4 m\} /\{1\}$ for $m \geq 0$.

Proof. The assertion (i) follows from (5) and Lemma 4.1 . The assertion (ii) follows from

$$
\begin{aligned}
\alpha_{m, 2 m} & =\sum_{k=0}^{0}(-1)^{k}\left[\begin{array}{c}
2 m+1 \\
k
\end{array}\right]\left[\begin{array}{c}
4 m-2 k+1 \\
4 m+1
\end{array}\right] \\
& =\left[\begin{array}{c}
2 m+1 \\
0
\end{array}\right]\left[\begin{array}{c}
4 m+1 \\
4 m+1
\end{array}\right] \\
& =1 .
\end{aligned}
$$

The assertion (iii) follows from

$$
\begin{aligned}
\alpha_{m, 2 m-1} & =\sum_{k=0}^{0}(-1)^{k}\left[\begin{array}{c}
2 m+1 \\
k
\end{array}\right]\left[\begin{array}{c}
4 m-2 k \\
4 m-1
\end{array}\right] \\
& =\left[\begin{array}{c}
2 m+1 \\
0
\end{array}\right]\left[\begin{array}{c}
4 m \\
4 m-1
\end{array}\right] \\
& =\left[\begin{array}{c}
4 m \\
1
\end{array}\right] \\
& =\{4 m\} /\{1\} .
\end{aligned}
$$

This completes the proof.

Proposition 4.3 implies the following.

Corollary 4.4. We have

(i) $x_{m, m}^{(n)}=0$ unless $\frac{n}{2} \leq m \leq 2 n$,

(ii) $x_{m, m}^{(2 m)}=(-1)^{m}\{2 m\}$ ! for $m \geq 0$, and

(iii) $x_{m, m}^{(2 m-1)}=(-1)^{m}\{2 m-1\} !\{4 m\} /\{1\}$ for $m \geq 0$.

\subsection{Divisibility property with respect to the cyclotomic polyno- mials}

Let us study the divisibility property of $\alpha_{m, n}$ and $x_{n, n}^{(m)}$ with respect to the cyclotomic polynomials. In what follows, we use also the symmetric version of the cyclotomic polynomial $\tilde{\Phi}_{l}=\prod_{d \mid l}\left(q^{d / 2}-q^{-d / 2}\right)^{\mu\left(\frac{l}{d}\right)} \in \mathbb{Z}\left[q^{1 / 2}, q^{-1 / 2}\right]$ for $l \geq 1$. For $f \in \mathbb{Z}\left[q^{1 / 2}, q^{-1 / 2}\right]$, $f \neq 0$, let $d_{l}(f)$ be the largest integer $i$ such that $f \in \tilde{\Phi}_{l}^{i} \mathbb{Z}\left[q^{1 / 2}, q^{-1 / 2}\right]$. 
Lemma 4.5. For $l \geq 1$ and $n \geq 0$, we have

$$
d_{l}(\{n\} !)=\left\lfloor\frac{n}{l}\right\rfloor .
$$

Proof. The assertion follows from

$$
d_{l}(\{i\})=d_{l}\left(q^{i / 2}-q^{-i / 2}\right)= \begin{cases}1 & \text { if } l \mid i \\ 0 & \text { otherwise }\end{cases}
$$

for $i \geq 0$.

Lemma 4.5 implies the following result.

Corollary 4.6. For $l \geq 1$ and $m, n \geq 0$, we have

$$
d_{l}\left(x_{m, m}^{(n)}\right)=\left\lfloor\frac{n}{l}\right\rfloor+d_{l}\left(\alpha_{m, n}\right) .
$$

Lemmas 4.1 and 4.5 imply the following result.

Corollary 4.7. For $l \geq 1$ and $m, n \geq 0$, we have

$$
d_{l}\left(\alpha_{m, n}\right)=\left\lfloor\frac{2 m+1}{l}\right\rfloor-\left\lfloor\frac{2 n+1}{l}\right\rfloor+d_{l}\left(\alpha_{n, m}\right) .
$$

In view of Corollaries 4.6 and 4.7 , in what follows, we study $d_{l}\left(\alpha_{m, n}\right)$ for $0 \leq m \leq n$. With respect to $\tilde{\Phi}_{1}$, we have the following result.

Proposition 4.8. For $0 \leq m \leq n \leq 2 m$, we have $d_{1}\left(\alpha_{m, n}\right)=0$.

We prove Proposition 4.8 by using the following lemma.

Lemma 4.9. For $0 \leq j \leq m$, we have

$$
\left.\alpha_{m, 2 m-j}\right|_{q^{1 / 2}=1}=4^{j}\left(\begin{array}{c}
m \\
j
\end{array}\right) .
$$

Proof of Proposition 4.8 assuming Lemma 4.9. By Lemma 4.9, for $0 \leq m \leq n \leq 2 m$, we have $\alpha_{m, n} \neq 0$ and $\left.\alpha_{m, n}\right|_{q^{1 / 2}=1} \neq 0$, which implies $d_{1}\left(\alpha_{m, n}\right)=0$.

Proof of Lemma 4.9. Set $\tilde{\alpha}_{m, j}=\left.\alpha_{m, 2 m-j}\right|_{q^{1 / 2}=1}$. It is enough to prove

$$
j \tilde{\alpha}_{m, j}-4(m-j+1) \tilde{\alpha}_{m, j-1}=0,
$$

which implies

$$
\begin{aligned}
\tilde{\alpha}_{m, j} & =4 \frac{(m-j+1)}{j} \tilde{\alpha}_{m, j-1} \\
& =\cdots=4^{j} \frac{(m-j+1)(m-j+2) \cdots(m)}{j(j-1) \cdots 1} \tilde{\alpha}_{m, 0}=4^{j}\left(\begin{array}{c}
m \\
j
\end{array}\right),
\end{aligned}
$$


where $\tilde{\alpha}_{m, 0}=1$ by Proposition 4.3 (ii).

We prove ([6]). Note that

$$
\tilde{\alpha}_{m, j}=\left.\alpha_{m, 2 m-j}\right|_{q^{1 / 2}=1}=\sum_{k=0}^{\left\lfloor m-\frac{2 m-j}{2}\right\rfloor} F(m, 2 m-j, k)=\sum_{k=0}^{\left\lfloor\frac{j}{2}\right\rfloor} \tilde{F}(m, j, k),
$$

with

$$
\begin{aligned}
F(m, n, k) & =(-1)^{k}\left(\begin{array}{c}
2 m+1 \\
k
\end{array}\right)\left(\begin{array}{c}
2 m+n-2 k+1 \\
2 n+1
\end{array}\right), \\
\tilde{F}(m, j, k) & =F(m, 2 m-j, k) .
\end{aligned}
$$

If we can find $G(m, j, k) \in \mathbb{Q}$ for $0 \leq k \leq\left\lfloor\frac{j}{2}\right\rfloor+1$ such that

$$
j \tilde{F}(m, j, k)-4(m-j+1) \tilde{F}(m, j-1, k)=G(m, j, k+1)-G(m, j, k)
$$

and

$$
G(m, j, 0)=G\left(m, j,\left\lfloor\frac{j}{2}\right\rfloor+1\right)=0
$$

then (6) follows from

$$
j \tilde{\alpha}_{m, j}-4(m-j+1) \tilde{\alpha}_{m, j-1}=G\left(m, j,\left\lfloor\frac{j}{2}\right\rfloor+1\right)-G(m, j, 0)=0 .
$$

Actually we can find such $G(m, j, k)$ by using Zeilberger's algorithm [17 as follows.

$$
G(m, j, k)= \begin{cases}-\frac{2 k(2 k-4 m+j-3)(2 k-4 m+j-2)}{(4 m-2 j+2)(4 m-2 j+3)} \tilde{F}(m, j, k) & \text { for } 1 \leq k \leq\left\lfloor\frac{j}{2}\right\rfloor, \\ 0 & \text { for } k=0,\left\lfloor\frac{j}{2}\right\rfloor+1 .\end{cases}
$$

We can check (7) and (8) by straightforward calculations. Hence we have the assertion.

See Table 1 for the behavior of $d_{1}\left(\alpha_{m, n}\right)$, where we color the boxes gray for $0 \leq n<m$ and the blanks mean $\alpha_{m, n}=0$.

For $l \geq 2$, we can compute $d_{l}\left(\alpha_{m, n}\right)$ for small $m, n \geq 0$ though we do not have a general result. See Table 2 for $d_{l}\left(\alpha_{m, n}\right)$ for $l=2, \ldots, 5$.

We have the following conjecture, which enables us to compute $d_{l}\left(\alpha_{m, n}\right)$ for general $m, n \geq 0$ by using explicit computations of $d_{l}\left(\alpha_{m, n}\right)$ for small $m, n \geq 0$.

Conjecture 4.10. For $l \geq 1$, in the range $0 \leq m \leq n \leq 2 m, d_{l}\left(\alpha_{m, n}\right)$ is periodic with period $l$ both in $m$ and in $n$, i.e., for $m \equiv \tilde{m}(\bmod l)$, and $n \equiv \tilde{n}(\bmod l)$, we have

$$
d_{l}\left(\alpha_{m, n}\right)=d_{l}\left(\alpha_{\tilde{m}, \tilde{n}}\right) .
$$

We also have the following conjecture.

Conjecture 4.11. For a prime $l \geq 1$ and $0 \leq m \leq n \leq 2 m$, we have $d_{l}\left(\alpha_{m, n}\right) \in\{0,1\}$. 


\begin{tabular}{|c|c|c|c|c|c|c|c|c|c|c|c|}
\hline$m \backslash n$ & 0 & 1 & 2 & 3 & 4 & 5 & 6 & 7 & 8 & 9 & 10 \\
\hline 0 & 0 & & & & & & & & & & \\
\hline 1 & & 0 & 0 & & & & & & & & \\
\hline 2 & & 2 & 0 & 0 & 0 & & & & & & \\
\hline 3 & & & 2 & 0 & 0 & 0 & 0 & & & & \\
\hline 4 & & & 4 & 2 & 0 & 0 & 0 & 0 & 0 & & \\
\hline 5 & & & & 4 & 2 & 0 & 0 & 0 & 0 & 0 & 0 \\
\hline 6 & & & & 6 & 4 & 2 & 0 & 0 & 0 & 0 & 0 \\
\hline 7 & & & & & 6 & 4 & 2 & 0 & 0 & 0 & 0 \\
\hline 8 & & & & & 8 & 6 & 4 & 2 & 0 & 0 & 0 \\
\hline
\end{tabular}

Table 1: $d_{1}\left(\alpha_{m, n}\right)$

\begin{tabular}{|c|c|c|c|c|c|c|c|c|c|c|c|}
\hline$m \backslash n$ & 0 & 1 & 2 & 3 & 4 & 5 & 6 & 7 & 8 & 9 & 10 \\
\hline 0 & 0 & & & & & & & & & & \\
\hline 1 & & 1 & 0 & & & & & & & & \\
\hline 2 & & 1 & 0 & 1 & 0 & & & & & & \\
\hline 3 & & & 2 & 1 & 0 & 1 & 0 & & & & \\
\hline 4 & & & 2 & 1 & 0 & 1 & 0 & 1 & 0 & & \\
\hline 5 & & & & 3 & 2 & 1 & 0 & 1 & 0 & 1 & 0 \\
\hline 6 & & & & 3 & 2 & 1 & 0 & 1 & 0 & 1 & 0 \\
\hline 7 & & & & & 4 & 3 & 2 & 1 & 0 & 1 & 0 \\
\hline 8 & & & & & 4 & 3 & 2 & 1 & 0 & 1 & 0 \\
\hline
\end{tabular}

Table 2: $d_{2}\left(\alpha_{m, n}\right)$

\begin{tabular}{|c|c|c|c|c|c|c|c|c|c|c|c|c|c|c|}
\hline$m \backslash n$ & 0 & 1 & 2 & 3 & 4 & 5 & 6 & 7 & 8 & 9 & 10 & 11 & 12 & 13 \\
\hline 0 & 0 & & & & & & & & & & & & & \\
\hline 1 & & 0 & 0 & & & & & & & & & & & \\
\hline 2 & & 0 & 0 & 0 & 0 & & & & & & & & & \\
\hline 3 & & & 1 & 0 & 1 & 1 & 0 & & & & & & & \\
\hline 4 & & & 2 & 2 & 0 & 0 & 1 & 0 & 0 & & & & & \\
\hline 5 & & & & 2 & 0 & 0 & 0 & 0 & 0 & 0 & 0 & & & \\
\hline 6 & & & & 2 & 2 & 1 & 0 & 1 & 1 & 0 & 1 & 1 & 0 & \\
\hline 7 & & & & & 2 & 2 & 2 & 0 & 0 & 1 & 0 & 0 & 1 & 0 \\
\hline 8 & & & & & 2 & 2 & 2 & 0 & 0 & 0 & 0 & 0 & 0 & 0 \\
\hline
\end{tabular}

Table 3: $d_{3}\left(\alpha_{m, n}\right)$ 


\begin{tabular}{|c|c|c|c|c|c|c|c|c|c|c|c|c|c|c|c|c|}
\hline$m \backslash n$ & 0 & 1 & 2 & 3 & 4 & 5 & 6 & 7 & 8 & 9 & 10 & 11 & 12 & 13 & 14 & 15 \\
\hline 0 & 0 & & & & & & & & & & & & & & & \\
\hline 1 & & 1 & 0 & & & & & & & & & & & & & \\
\hline 2 & & 1 & 0 & 1 & 0 & & & & & & & & & & & \\
\hline 3 & & & 1 & 1 & 0 & 1 & 0 & & & & & & & & & \\
\hline 4 & & & 1 & 1 & 0 & 2 & 1 & 1 & 0 & & & & & & & \\
\hline 5 & & & & 2 & 2 & 1 & 0 & 2 & 1 & 1 & 0 & & & & & \\
\hline 6 & & & & 2 & 2 & 1 & 0 & 1 & 0 & 1 & 0 & 1 & 0 & & & \\
\hline 7 & & & & & 2 & 3 & 1 & 1 & 0 & 1 & 0 & 1 & 0 & 1 & 0 & \\
\hline 8 & & & & & 2 & 3 & 1 & 1 & 0 & 2 & 1 & 1 & 0 & 2 & 1 & 1 \\
\hline
\end{tabular}

Table 4: $d_{4}\left(\alpha_{m, n}\right)$

\begin{tabular}{|c|c|c|c|c|c|c|c|c|c|c|c|c|c|c|c|c|}
\hline$m \backslash n$ & 0 & 1 & 2 & 3 & 4 & 5 & 6 & 7 & 8 & 9 & 10 & 11 & 12 & 13 & 14 & 15 \\
\hline 0 & 0 & & & & & & & & & & & & & & & \\
\hline 1 & & 0 & 0 & & & & & & & & & & & & & \\
\hline 2 & & 1 & 0 & 0 & 0 & & & & & & & & & & & \\
\hline 3 & & & 0 & 0 & 0 & 0 & 0 & & & & & & & & & \\
\hline 4 & & & 0 & 0 & 0 & 0 & 0 & 0 & 0 & & & & & & & \\
\hline 5 & & & & 1 & 1 & 0 & 1 & 1 & 1 & 1 & 0 & & & & & \\
\hline 6 & & & & 1 & 1 & 1 & 0 & 0 & 1 & 1 & 1 & 0 & 0 & & & \\
\hline 7 & & & & & 2 & 2 & 1 & 0 & 0 & 0 & 1 & 1 & 0 & 0 & 0 & \\
\hline 8 & & & & & 2 & 2 & 0 & 0 & 0 & 0 & 0 & 0 & 0 & 0 & 0 \\
\hline 9 & & & & & & 2 & 2 & 0 & 0 & 0 & 0 & 0 & 0 & 0 & 0 & 0 \\
\hline 10 & & & & & 2 & 3 & 2 & 1 & 1 & 0 & 1 & 1 & 1 & 1 & 0 \\
\hline
\end{tabular}

Table 5: $d_{5}\left(\alpha_{m, n}\right)$ 


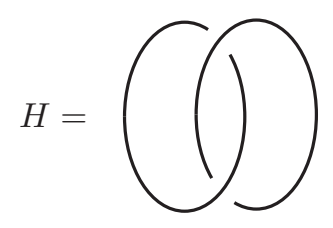

Figure 3: The Hopf link $H$

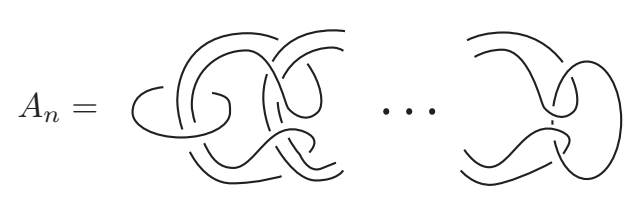

Figure 4: Milnor's link $A_{n}$

\section{Colored Jones polynomial of Milnor's link}

In this section, we give examples of computations of the reduced colored Jones polynomials of Milnor's link.

For $n \geq 3$, let $A_{n}$ be the $n$-component Milnor's link depicted in Figure 4 Let $A_{2}=H$ be the Hopf link depicted in Figure 3. Note that $A_{3}=B$ is the Borromean rings, and $A_{n}=B\left(A_{n-1} ; 1\right)$ for $n \geq 3$.

We use the following result.

Lemma 5.1 (Habiro [12, Corollary 14.2]). For $i, j, k \geq 0$, we have

$$
J_{B ; P_{i}^{\prime}, P_{j}^{\prime}, P_{k}^{\prime}}= \begin{cases}(-1)^{i}\{2 i+1\}_{i+1} /\{1\} & \text { if } i=j=k, \\ 0 & \text { otherwise. }\end{cases}
$$

By Theorem 3.2 and Lemma 5.1 we can compute the reduced colored Jones polynomial of $A_{n}$ as follows.

Proposition 5.2. For $n \geq 3$, we have

$$
\begin{aligned}
J_{A_{n} ; P_{l_{1}}^{\prime}, \ldots, P_{l_{n}}^{\prime}} & =\delta_{l_{1}, l_{2}} \delta_{l_{n-1}, l_{n}} x_{l_{2}, l_{2}}^{\left(l_{3}\right)} x_{l_{3}, l_{3}}^{\left(l_{4}\right)} \cdots x_{l_{n-2}, l_{n-2}}^{\left(l_{n-1}\right)} J_{B ; P_{l_{n-1}}^{\prime}, P_{l_{n-1}}^{\prime}, P_{l_{n-1}}^{\prime}} \\
& =\delta_{l_{1}, l_{2}} \delta_{l_{n-1}, l_{n}} x_{l_{2}, l_{2}}^{\left(l_{3}\right)} x_{l_{3}, l_{3}}^{\left(l_{4}\right)} \cdots x_{l_{n-2}, l_{n-2}}^{\left(l_{n-1}\right)}(-1)^{l_{n-1}}\left\{2 l_{n-1}+1\right\}_{l_{n-1}+1} /\{1\} .
\end{aligned}
$$

Proof. We use induction on $n$. For $n=3$, by Lemma 5.1, we have

$$
J_{B ; P_{l_{1}}^{\prime}, P_{l_{2}}^{\prime}, P_{l_{3}}^{\prime}}=\delta_{l_{1}, l_{2}} \delta_{l_{2}, l_{3}} J_{B ; P_{l_{2}}^{\prime}, P_{l_{2}}^{\prime}, P_{l_{2}}^{\prime}} .
$$


For $n>3$, by the assumption of induction, we have

$$
\begin{aligned}
J_{A_{n} ; P_{l_{1}}^{\prime}, \ldots, P_{l_{n}}^{\prime}} & =\sum_{k_{1} \geq 0} x_{l_{1}, l_{2}}^{\left(k_{1}\right)} J_{A_{n-1} ; P_{k_{1}}^{\prime}, P_{l_{3}}^{\prime}, \ldots, P_{l_{n}}^{\prime}} \\
& =\delta_{l_{1}, l_{2}} \sum_{k_{1} \geq 0} x_{l_{2}, l_{2}}^{\left(k_{1}\right)} J_{A_{n-1} ; P_{k_{1}}^{\prime}, P_{l_{3}}^{\prime}, \ldots, P_{l_{n}}^{\prime}} \\
& =\delta_{l_{1}, l_{2}} \sum_{k_{1} \geq 0} x_{l_{2}, l_{2}}^{\left(k_{1}\right)} \delta_{k_{1}, l_{3}} \delta_{l_{n-1}, l_{n}} x_{l_{3}, l_{3}}^{\left(l_{4}\right)} x_{l_{4}, l_{4}}^{\left(l_{5}\right)} \cdots x_{l_{n-2}, l_{n-2}}^{\left(l_{n-1}\right)} J_{A ; P_{l_{n-1}}^{\prime}, P_{l_{n-1}}^{\prime}, P_{l_{n-1}}^{\prime}} \\
& =\delta_{l_{1}, l_{2}} \delta_{l_{n-1}, l_{n}} x_{l_{1}, l_{2}}^{\left(l_{3}\right)} x_{l_{3}, l_{3}}^{\left(l_{4}\right)} x_{l_{4}, l_{4}}^{\left(l_{5}\right)} \cdots x_{l_{n-2}, l_{n-2}}^{\left(l_{n-1}\right)} J_{A ; P_{l_{n-1}}^{\prime}, P_{l_{n-1}}^{\prime}, P_{l_{n-1}}^{\prime}} .
\end{aligned}
$$

Thus we have the assertion.

We have the following corollaries.

Corollary 5.3. For $n \geq 3$, we have

$$
J_{A_{n} ; P_{1}^{\prime}, \ldots, P_{1}^{\prime}}=(-1)^{n} \tilde{\Phi}_{1}^{n-2} \tilde{\Phi}_{2}^{n-2} \tilde{\Phi}_{3} \tilde{\Phi}_{4}^{n-3} .
$$

Proof. The assertion follows from Proposition 5.2 and

$$
x_{1,1}^{(1)}=-\{4\}=-\tilde{\Phi}_{4} \tilde{\Phi}_{2} \tilde{\Phi}_{1}
$$

and

$$
J_{B ; P_{1}^{\prime}, P_{1}^{\prime}, P_{1}^{\prime}}=-\{3\}_{2} /\{1\}=-\tilde{\Phi}_{3} \tilde{\Phi}_{2} \tilde{\Phi}_{1}
$$

Corollary 5.4. (i) For $n \geq 3$ and $l_{1}, \ldots, l_{n} \geq 0$, unless $l_{1}=l_{2}, l_{n-1}=l_{n}$ and unless $\frac{1}{2} \leq \frac{l_{i+1}}{l_{i}} \leq 2$ for $2 \leq i \leq n-2$, we have $J_{A_{n} ; P_{l_{1}}^{\prime}, \ldots, P_{l_{n}}^{\prime}}=0$.

(ii) For $a_{0} \geq 0$ and $a_{i}=2 a_{i-1}$ for $i=1, \ldots, n-3$, we have

$$
J_{A_{n} ; P_{a_{0}}^{\prime}, P_{a_{0}}^{\prime}, P_{a_{1}}^{\prime}, \ldots, P_{a_{n-4}}^{\prime}, P_{a_{n-3}}^{\prime}, P_{a_{n-3}}^{\prime}}=\left(\prod_{m=1}^{n-3}(-1)^{a_{m}}\left\{2 a_{m}\right\} !\right)\left((-1)^{a_{n-3}}\left\{2 a_{n-3}+1\right\}_{a_{n-3}+1} /\{1\}\right) .
$$

(iii) For $b_{0} \geq 0$ and $b_{i}=2 b_{i-1}-1$ for $i=1, \ldots, n-3$ (i.e., $\left.b_{i}=2^{i} b_{0}-\frac{i(i+1)(2 i+1)}{6}\right)$ we have

$$
\begin{aligned}
& J_{A_{n} ; P_{b_{0}}^{\prime}, P_{b_{0}}^{\prime}, P_{b_{1}}^{\prime}, \ldots, P_{b_{n-4}}^{\prime}, P_{b_{n-3}}^{\prime}, P_{b_{n-3}}^{\prime}} \\
& =\left(\prod_{m=1}^{n-3}(-1)^{b_{m}}\left\{2 b_{m}-1\right\} !\left\{4 b_{m}\right\} /\{1\}\right)\left((-1)^{b_{n-3}}\left\{2 b_{n-3}+1\right\}_{b_{n-3}+1} /\{1\}\right) .
\end{aligned}
$$

Proof. The assertions (i), (ii) and (iii) follow from Corollary 4.4 (i), (ii) and (iii), respectively, and Proposition 5.2 


\section{Proofs}

In this section, we prove Theorems 3.2 6.5, and Proposition 3.8 .

\subsection{Proof of Theorem 3.2}

We prove Theorem 3.2. We also prove Lemma 4.1 at the end of this section.

In [12, Habiro defined the element

$$
S_{n}=\prod_{i=1}^{n}\left(V_{2}-\left(q^{i}+1+q^{-i}\right)\right) \in \mathcal{R},
$$

for $n \geq 0$, which is a kind of dual of $P_{n}^{\prime}$ with respect to the symmetric bilinear form $J_{H ;-,-}: \mathcal{R} \times \mathcal{R} \rightarrow \mathbb{Q}\left(q^{1 / 2}\right)$ as follows.

Lemma 6.1 (Habiro [12, Proposition 6.6]). For $m, n \geq 0$, we have

$$
J_{H ; P_{m}^{\prime}, S_{n}}=\delta_{m, n}\{2 m+1\}_{2 m} /\{m\} !
$$

Recall the element $\alpha_{m, n}=\alpha_{m, n}\left(q^{1 / 2}\right) \in \mathbb{Z}\left[q^{1 / 2}, q^{-1 / 2}\right]$ defined in Theorem 3.2 (ii). We reduce Theorem 3.2 to the following proposition.

Proposition 6.2. For $l \geq 0$, we have

$$
S_{l}=\sum_{m \geq 0} \alpha_{l, m}\{m\} ! P_{m}^{\prime}
$$

Proof of Theorem 3.2 assuming Proposition 6.2. By Proposition 6.2 and Lemma5.1 we have

$$
\begin{aligned}
J_{B ; P_{i}^{\prime}, P_{j}^{\prime}, S_{l}} & =\sum_{m \geq 0} \alpha_{l, m}\{m\} ! J_{B ; P_{i}^{\prime}, P_{j}^{\prime}, P_{m}^{\prime}} \\
& =\delta_{i, j} \alpha_{l, i}\{i\} !(-1)^{i}\{2 i+1\}_{i+1} /\{1\} \\
& =\delta_{i, j} \alpha_{l, i}(-1)^{i}\{2 i+1\} ! /\{1\} .
\end{aligned}
$$

On the other hand, since $B=B(H ; 1)$, we have

$$
\begin{aligned}
J_{A ; P_{i}^{\prime}, P_{j}^{\prime}, S_{l}} & =J_{B(H ; 1) ; P_{i}^{\prime}, P_{j}^{\prime}, S_{l}} \\
& =\sum_{k \geq 0} x_{i, j}^{(k)} J_{H ; P_{k}^{\prime}, S_{l}} \\
& =x_{i, j}^{(l)}\{2 l+1\}_{2 l} /\{l\} ! .
\end{aligned}
$$

Here, the last identity follows from Lemma 6.1

Consequently, we have

$$
x_{i, j}^{(l)}=\delta_{i, j}(-1)^{i} \frac{\{2 i+1\} !\{l\} !}{\{2 l+1\} !} \alpha_{l, i},
$$

which completes the proof. 
In what follows, we prove Proposition 6.2. We use two more lemmas as follows.

Lemma 6.3. For $l \geq 0$, we have

$$
S_{l}=\sum_{k=0}^{l}(-1)^{k}\left[\begin{array}{c}
2 l+1 \\
k
\end{array}\right] V_{2 l-2 k} .
$$

Proof. We use an induction on $l$. For $m=0,1$, we have

$$
\begin{aligned}
& S_{0}=1, \\
& S_{1}=\left(V_{2}-\left(q+1+q^{-1}\right)\right)=V_{2}-[3] .
\end{aligned}
$$

For $m \geq 2$, we have

$$
\begin{aligned}
S_{m}= & S_{m-1}\left(V_{2}-\left(q^{m}+1+q^{-m}\right)\right) \\
= & \sum_{k=0}^{m-1}(-1)^{k}\left[\begin{array}{c}
2 m-1 \\
k
\end{array}\right] V_{2 m-2 k-2}\left(V_{2}-\left(q^{m}+1+q^{-m}\right)\right) \\
= & \sum_{k=0}^{m-2}(-1)^{k}\left[\begin{array}{c}
2 m-1 \\
k
\end{array}\right]\left(V_{2 m-2 k}+V_{2 m-2 k-2}+V_{2 m-2 k-4}-\left(q^{m}+1+q^{-m}\right) V_{2 m-2 k-2}\right) \\
& +(-1)^{m-1}\left[\begin{array}{c}
2 m-1 \\
m-1
\end{array}\right]\left(V_{2}-\left(q^{m}+1+q^{-m}\right)\right) \\
= & \sum_{k=0}^{m-2}(-1)^{k}\left[\begin{array}{c}
2 m-1 \\
k
\end{array}\right]\left(V_{2 m-2 k}-\left(q^{m}+q^{-m}\right) V_{2 m-2 k-2}+V_{2 m-2 k-4}\right) \\
& +(-1)^{m-1}\left[\begin{array}{c}
2 m-1 \\
m-1
\end{array}\right]\left(V_{2}-\left(q^{m}+1+q^{-m}\right)\right) \\
= & V_{2 m}-\left(q^{m}+q^{-m}\right) V_{2 m-2}-[2 m-1] V_{2 m-2} \\
& +\sum_{k=2}^{m-1}\left((-1)^{k-2}\left[\begin{array}{c}
2 m-1 \\
k-2
\end{array}\right]-(-1)^{k-1}\left[\begin{array}{c}
2 m-1 \\
k-1
\end{array}\right]\left(q^{m}+q^{-m}\right)+(-1)^{k}\left[\begin{array}{c}
2 m-1 \\
k
\end{array}\right]\right) V_{2 m-2 k} \\
& +(-1)^{m-2}\left[\begin{array}{c}
2 m-1 \\
m-2
\end{array}\right]-(-1)^{m-1}\left[\begin{array}{c}
2 m-1 \\
m-1
\end{array}\right]\left(q^{m}+1+q^{-m}\right) \\
= & \sum_{k=1}^{m}(-1)^{k}\left[\begin{array}{c}
2 m+1 \\
k
\end{array}\right] V_{2 m-2 k} .
\end{aligned}
$$

The following lemma is observed in the proof of [12, Proposition 6.6].

Lemma 6.4 (Habiro [12]). For $m, n \geq 0$, we have

$$
J_{H ; V_{m}, S_{n}}=\{m+n+1\}_{2 n+1} /\{1\} .
$$

Now, we prove Proposition 6.2 
Proof of Proposition 6.2. For $m, n \geq 0$, we have

$$
\begin{aligned}
J_{H ; S_{m}, S_{n}} & =\sum_{l \geq 0} \alpha_{m, l}\{l\} ! J_{H ; P_{l}^{\prime}, S_{n}} \\
& =\alpha_{m, n}\{n\} !\left(\{2 n+1\}_{2 n} /\{n\} !\right) \\
& =\alpha_{m, n}\{2 n+1\}_{2 n} .
\end{aligned}
$$

Hence we have

$$
\begin{aligned}
\alpha_{m, n} & =J_{H ; S_{m}, S_{n}} /\{2 n+1\}_{2 n} \\
& =\sum_{k=1}^{m}(-1)^{k}\left[\begin{array}{c}
2 m+1 \\
k
\end{array}\right] J_{H ; V_{2 m-2 k}, S_{n}} /\{2 n+1\}_{2 n} \\
& =\sum_{k=1}^{m}(-1)^{k}\left[\begin{array}{c}
2 m+1 \\
k
\end{array}\right]\{2 m+n-2 k+1\}_{2 n+1} /\{2 n+1\} ! \\
& =\sum_{k=1}^{m}(-1)^{k}\left[\begin{array}{c}
2 m+1 \\
k
\end{array}\right]\left[\begin{array}{c}
2 m+n-2 k+1 \\
2 n+1
\end{array}\right],
\end{aligned}
$$

where the second identity follows from Lemma 6.3 and the third identity follows from Lemma 6.4. Hence we have the assertion.

We prove Lemma 4.1 .

Proof of Lemma 4.1. By (11) and symmetric property of $J_{H ; S_{m}, S_{n}}$, we have

$$
\begin{aligned}
J_{H ; S_{m}, S_{n}} & =\alpha_{m, n}\{2 n+1\}_{2 n} \\
& =\alpha_{n, m}\{2 m+1\}_{2 m}
\end{aligned}
$$

for $m, n \geq 0$. Thus we have

$$
\begin{aligned}
\alpha_{m, n} & =\frac{\{2 m+1\}_{2 m}}{\{2 n+1\}_{2 n}} \alpha_{n, m} \\
& =\frac{\{2 m+1\} !}{\{2 n+1\} !} \alpha_{n, m} .
\end{aligned}
$$

Hence we have the assertion.

\subsection{Proof of Theorem 3.4}

We reduce Theorem 3.4 to Proposition 6.5 as follows.

Proposition 6.5. Let $L=L_{1} \cup \cdots \cup L_{n}$ be an algebraically-split, 0-framed link in $S^{3}$ and set $\check{L}=L_{2} \cup L_{3} \cup \cdots \cup L_{n}$. For $\epsilon, \epsilon^{\prime}, \epsilon_{2}, \ldots, \epsilon_{n} \in\{ \pm 1\}$, we have

$$
J_{S^{3}\left(B(L ; 1) ; \epsilon, \epsilon^{\prime}, \epsilon_{2}, \ldots, \epsilon_{n}\right)}-J_{S^{3}\left(\check{L} ; \epsilon_{2}, \ldots, \epsilon_{n}\right)} \in \Phi_{1}^{2} \Phi_{2}^{2} \Phi_{3} \Phi_{4} \Phi_{6} \widehat{\mathbb{Z}[q]} .
$$


Proof of Theorem 3.4 assuming Proposition 6.5. Let $M$ and $M^{\prime}$ be integral homology spheres related by a special Bing double surgery, i.e., $M^{\prime}$ is orientation-preserving homeomorphic to $M\left(B(K) ; \epsilon, \epsilon^{\prime}\right)$ for a 0 -framed knot $K$ and $\epsilon, \epsilon^{\prime} \in\{ \pm 1\}$. Let $T=T_{1} \cup \cdots \cup T_{n}$ be an algebraically split link in $S^{3}$ such that $S^{3}\left(T ; \epsilon_{1}, \ldots, \epsilon_{n}\right), \epsilon_{1}, \ldots, \epsilon_{n} \in\{ \pm 1\}$, is orientation-preserving homeomorphic to $M$. Here, by isotopy of $K$ in $M$, we can assume that $K$ is null-homologous in $M \backslash N$, where $N$ is the union of solid tori on which the surgery operation along $T$ was done. Now, $K$ may be regarded as a 0 -framed knot in $S^{3} \backslash T$. Set $L=K \cup T$. By Proposition 6.5 we have

$$
\begin{aligned}
J_{M\left(B(K) ; \epsilon, \epsilon^{\prime}\right)}-J_{M} & =J_{S^{3}\left(B(L ; 1) ; \epsilon, \epsilon^{\prime}, \epsilon_{1}, \ldots, \epsilon_{n}\right)}-J_{S^{3}\left(\check{L}=T ; \epsilon_{1}, \ldots, \epsilon_{n}\right)} \\
& \in \Phi_{1}^{2} \Phi_{2}^{2} \Phi_{3} \Phi_{4} \Phi_{6} \widehat{\mathbb{Z}[q]} .
\end{aligned}
$$

Hence we have the assertion.

We prove Proposition 6.5. We use the following lemma.

Lemma 6.6 (Habiro [12, Theorem 8.2]). Let L be an $n$-component, algebraically-split link with 0 -framing. For $l_{1}, \ldots, l_{n} \geq 0$, we have

$$
J_{L ; P_{l_{1}}^{\prime}, \ldots, P_{l_{n}}^{\prime}} \in \frac{\left\{2 l_{j}+1\right\}_{l_{j}+1}}{\{1\}} \mathbb{Z}\left[q^{1 / 2}, q^{-1 / 2}\right],
$$

where $j$ is an integer such that $l_{j}=\max \left\{l_{i}\right\}_{1 \leq i \leq n}$.

Proof of Proposition 6.5. Let $L$ be an $n$-component, algebraically-split link with 0 framing. By the definition, we have

$$
\begin{aligned}
& J_{S^{3}\left(B(L ; 1) ; \epsilon, \epsilon^{\prime}, \epsilon_{2}, \ldots, \epsilon_{n}\right)}=J_{B(L ; 1) ; \omega-\epsilon, \omega}-\epsilon^{\prime}, \omega-\epsilon_{2}, \ldots, \omega^{-\epsilon_{n}} \\
& =\sum_{i \geq 0} \sum_{j \geq 0}(-\epsilon)^{i}\left(-\epsilon^{\prime}\right)^{j} q^{-\epsilon i(i+3) / 4-\epsilon^{\prime} j(j+3) / 4} J_{B(L ; 1) ; P_{i}^{\prime}, P_{j}^{\prime}, \omega^{-\epsilon_{2}, \ldots, \omega}, \omega_{n} .} .
\end{aligned}
$$

By Lemma 3.1 and Theorem 3.2 we have

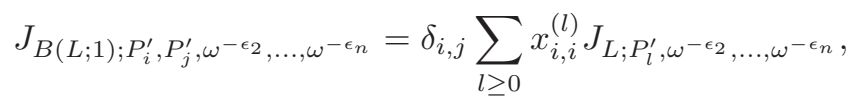

thus we have

$$
\begin{aligned}
& \sum_{i \geq 0} \sum_{j \geq 0}(-\epsilon)^{i}\left(-\epsilon^{\prime}\right)^{j} q^{-\epsilon i(i+3) / 4-\epsilon^{\prime} j(j+3) / 4} J_{B(L ; 1) ; P_{i}^{\prime}, P_{j}^{\prime}, \omega^{-\epsilon_{2}, \ldots, \omega}, \omega_{n}}
\end{aligned}
$$

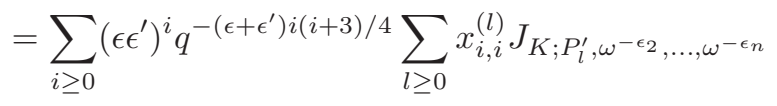

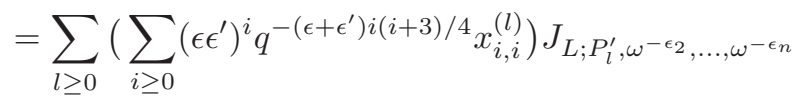

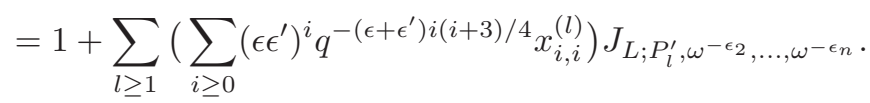


For $l \geq 1$, set

$$
\begin{aligned}
s_{l}^{\left(\epsilon, \epsilon^{\prime}\right)} & =\sum_{i \geq 0}\left(\epsilon \epsilon^{\prime}\right)^{i} q^{-\left(\epsilon+\epsilon^{\prime}\right) i(i+3) / 4} x_{i, i}^{(l)} \\
& =\sum_{i \geq\lceil l / 2\rceil}^{2 l}\left(\epsilon \epsilon^{\prime}\right)^{i} q^{-\left(\epsilon+\epsilon^{\prime}\right) i(i+3) / 4} x_{i, i}^{(l)},
\end{aligned}
$$

where the second identity follows from Corollary 4.4 (i). It is enough to prove

$$
s_{l}^{\left(\epsilon, \epsilon^{\prime}\right)} J_{L ; P_{l}^{\prime}, P_{l_{2}}^{\prime}, \ldots, P_{l_{n}}^{\prime}} \in \tilde{\Phi}_{1}^{2} \tilde{\Phi}_{2}^{2} \tilde{\Phi}_{3} \tilde{\Phi}_{4} \tilde{\Phi}_{6} \mathbb{Z}\left[q^{1 / 2}, q^{-1 / 2}\right],
$$

for $l \geq 1$ and $l_{2}, \ldots, l_{n} \geq 0$.

By Lemma 6.6. we have

$$
\begin{aligned}
& J_{L ; P_{1}^{\prime}, P_{l_{2}}^{\prime}, \ldots, P_{l_{n}}^{\prime}} \in \frac{\{3\}_{2}}{\{1\}} \mathbb{Z}\left[q^{1 / 2}, q^{-1 / 2}\right] \subset \tilde{\Phi}_{1} \tilde{\Phi}_{2} \tilde{\Phi}_{3} \mathbb{Z}\left[q^{1 / 2}, q^{-1 / 2}\right], \\
& J_{L ; P_{2}^{\prime}, P_{l_{2}}^{\prime}, \ldots, P_{l_{n}}^{\prime}} \in \frac{\{5\}_{3}}{\{1\}} \mathbb{Z}\left[q^{1 / 2}, q^{-1 / 2}\right] \subset \tilde{\Phi}_{1}^{2} \tilde{\Phi}_{2} \tilde{\Phi}_{3} \tilde{\Phi}_{4} \tilde{\Phi}_{5} \mathbb{Z}\left[q^{1 / 2}, q^{-1 / 2}\right],
\end{aligned}
$$

and for $l \geq 3$, we have

$$
\begin{aligned}
J_{L ; P_{l}^{\prime}, P_{l_{2}}^{\prime}, \ldots, P_{l_{n}}^{\prime}} & \in \frac{\{2 l+1\}_{l+1}}{\{1\}} \mathbb{Z}\left[q, q^{-1}\right] \\
& \subset \tilde{\Phi}_{1}^{2} \tilde{\Phi}_{2}^{2} \tilde{\Phi}_{3} \tilde{\Phi}_{4} \tilde{\Phi}_{6} \mathbb{Z}\left[q^{1 / 2}, q^{-1 / 2}\right] .
\end{aligned}
$$

Thus we have only to prove

$$
\begin{aligned}
& s_{1}^{\left(\epsilon, \epsilon^{\prime}\right)} \in \tilde{\Phi}_{1} \tilde{\Phi}_{2} \tilde{\Phi}_{4} \tilde{\Phi}_{6} \mathbb{Z}\left[q^{1 / 2}, q^{-1 / 2}\right], \\
& s_{2}^{\left(\epsilon, \epsilon^{\prime}\right)} \in \tilde{\Phi}_{2} \tilde{\Phi}_{6} \mathbb{Z}\left[q^{1 / 2}, q^{-1 / 2}\right] .
\end{aligned}
$$

By Corollaries 4.2 and 4.4 , we have

$$
\begin{aligned}
& x_{1,1}^{(1)}=-\{4\}=-\tilde{\Phi}_{1} \tilde{\Phi}_{2} \tilde{\Phi}_{4}, \\
& x_{2,2}^{(1)}=(-1)^{3} \frac{\{5\} !\{1\} !}{\{3\} !\{2\} !} x_{1,1}^{(2)}=\left((-1)^{3} \frac{\{5\} !\{1\} !}{\{3\} !\{2\} !}\right)(-\{2\} !)=\tilde{\Phi}_{1}^{3} \tilde{\Phi}_{2} \tilde{\Phi}_{4} \tilde{\Phi}_{5}, \\
& x_{1,1}^{(2)}=-\{2\} !=-\tilde{\Phi}_{1}^{2} \tilde{\Phi}_{2}, \\
& x_{2,2}^{(2)}=\tilde{\Phi}_{1}^{2} \tilde{\Phi}_{2}\left(q^{-5}+q^{-4}+2 q^{-3}+q^{-2}+2 q^{-1}+2+2 q+q^{2}+2 q^{3}+q^{4}+q^{5}\right), \\
& x_{2,2}^{(3)}=(-1)^{2}\{3\} !\{8\} /\{1\}=\tilde{\Phi}_{1}^{3} \tilde{\Phi}_{2}^{2} \tilde{\Phi}_{3} \tilde{\Phi}_{4} \tilde{\Phi}_{8}, \\
& x_{2,2}^{(4)}=\{4\} !=\tilde{\Phi}_{1}^{4} \tilde{\Phi}_{2}^{2} \tilde{\Phi}_{3} \tilde{\Phi}_{4} .
\end{aligned}
$$


Thus we have

$$
\begin{aligned}
s_{1}^{(-1,-1)} & =\sum_{i=1}^{2} q^{i(i+3) / 2} x_{i, i}^{(1)} \\
& =\Phi_{1} \Phi_{2} \Phi_{4} \Phi_{6} \cdot q\left(1-q+q^{3}\right), \\
s_{2}^{(-1,-1)} & =\sum_{i=1}^{4} q^{i(i+3) / 2} x_{i, i}^{(2)} \\
& =\Phi_{1}^{2} \Phi_{2} \Phi_{3} \Phi_{6} \cdot q^{5 / 2}\left(1+q+q^{2}+q^{3}+q^{4}+q^{5}+q^{6}-q^{13}-q^{14}-q^{16}+q^{21}\right), \\
s_{1}^{(1,1)} & =\sum_{i=1}^{2} q^{-i(i+3) / 2} x_{i, i}^{(1)} \\
& =-\Phi_{1} \Phi_{2} \Phi_{4} \Phi_{6} \cdot q^{-10}\left(-1+q^{2}+q^{3}\right), \\
s_{2}^{(1,1)} & =\sum_{i=1}^{4} q^{-i(i+3) / 2} x_{i, i}^{(2)} \\
& =\Phi_{1}^{2} \Phi_{2} \Phi_{3} \Phi_{6} \cdot q^{-61 / 2}\left(1-q^{2}-q^{7}-q^{8}+q^{15}+q^{16}+q^{17}+q^{18}+q^{19}+q^{20}+q^{21}\right), \\
s_{1}^{(-1,1)}=s_{1}^{(1,-1)} & =\sum_{i=1}^{2}(-1)^{i} x_{i, i}^{(1)} \\
& =\Phi_{1} \Phi_{2} \Phi_{4} \Phi_{6} \Phi_{12} \cdot q^{-5}, \\
s_{2}^{(-1,1)}=s_{2}^{(1,-1)} & =\sum_{n=1}^{4}(-1)^{n} x_{n, n}^{(2)} \\
& =\Phi_{1}^{2} \Phi_{2} \Phi_{6} \cdot q^{-33 / 2}\left(1+q-q^{3}-q^{4}+q^{5}+2 q^{6}+q^{7}-q^{8}-2 q^{9}+q^{10}+4 q^{11}+4 q^{12}\right. \\
& \left.-3 q^{14}+4 q^{16}+4 q^{17}+q^{18}-2 q^{19}-q^{20}+q^{21}+2 q^{22}+q^{23}-q^{24}-q^{25}+q^{27}+q^{28}\right) .
\end{aligned}
$$

Hence we have (12) and (13). This completes the proof.

\subsection{Proof of Proposition 3.8}

To prove Proposition 3.8 we use the following lemma.

Lemma 6.7 (Habiro[12, Proposition 14.5]). For $i, j, k \in \mathbb{Z}$, we have

$$
J_{M_{i, j, k}}=\sum_{l \geq 0} \omega_{i, l} \omega_{j, l} \omega_{k, l}(-1)^{l}\{2 l+1\}_{l+1} /\{1\}
$$

where for $p \in \mathbb{Z}$ and $n \geq 0$,

$$
\omega_{p, n}=\left\{\begin{array}{l}
q^{\frac{1}{4} n(n+3)} \sum_{\mathbf{i} \in S(n, p)}\left[\begin{array}{c}
n \\
\mathbf{i}
\end{array}\right]_{q} q^{f(\mathbf{i})} \quad \text { for } p \geq 0, \\
(-1)^{n} q^{-\frac{1}{4} n(n+3)} \sum_{\mathbf{i} \in S(n,-p)}\left[\begin{array}{c}
n \\
\mathbf{i}
\end{array}\right]_{q-1} q^{-f(\mathbf{i})} \quad \text { for } p \leq 0 .
\end{array}\right.
$$


Here for $p, n \geq 0$, we set

$$
S(n, p)=\left\{\left(i_{1}, \ldots, i_{p}\right) \mid i_{1}, \ldots, i_{p} \geq 0, i_{1}+\cdots+i_{p}=n\right\},
$$

and for $\mathbf{i}=\left(i_{1}, \ldots, i_{p}\right) \in S(n, p)$, we set

$$
\left[\begin{array}{c}
n \\
\mathbf{i}
\end{array}\right]_{q}=\frac{[n]_{q} !}{\left[i_{1}\right]_{q} ! \cdots\left[i_{p}\right]_{q} !}, \quad f(\mathbf{i})=\sum_{j=1}^{p-1}\left(s_{j}^{2}+s_{j}\right),
$$

where $s_{j}=\sum_{k=1}^{j} i_{k}$ and

$$
[m]_{q}=\frac{q^{m}-1}{q-1}, \quad[m]_{q} !=[m]_{q}[m-1]_{q} \cdots[1]_{q},
$$

for $m \geq 0$.

Proof of Proposition 3.8. By Lemma 6.7 and $\omega_{p, 0}=1$ for $p \in \mathbb{Z}$, we have

$$
\begin{aligned}
J_{M_{i, j, k}}-1 & =\sum_{l \geq 1} \omega_{i, l} \omega_{j, l} \omega_{k, l}(-1)^{l}\{2 l+1\}_{l+1} /\{1\} \\
& \equiv-\omega_{i, 1} \omega_{j, 1} \omega_{k, 1} \tilde{\Phi}_{3} \tilde{\Phi}_{2} \tilde{\Phi}_{1}+\omega_{i, 2} \omega_{j, 2} \omega_{k, 2} \tilde{\Phi}_{5} \tilde{\Phi}_{4} \tilde{\Phi}_{3} \tilde{\Phi}_{2} \tilde{\Phi}_{1}^{2} \quad\left(\bmod \tilde{\Phi}_{2}^{2}\right) \\
& \equiv-q^{-2} \omega_{i, 1} \omega_{j, 1} \omega_{k, 1} \Phi_{3} \Phi_{2} \Phi_{1}+q^{-5-\frac{1}{2}} \omega_{i, 2} \omega_{j, 2} \omega_{k, 2} \Phi_{5} \Phi_{4} \Phi_{3} \Phi_{2} \Phi_{1}^{2} \quad\left(\bmod \Phi_{2}^{2}\right) .
\end{aligned}
$$

Thus we have

$$
\frac{J_{M_{i, j, k}}-1}{\Phi_{2}}=-q^{-2} \omega_{i, 1} \omega_{j, 1} \omega_{k, 1} \Phi_{3} \Phi_{1}+q^{-5-\frac{1}{2}} \omega_{i, 2} \omega_{j, 2} \omega_{k, 2} \Phi_{5} \Phi_{4} \Phi_{3} \Phi_{1}^{2} \quad\left(\bmod \Phi_{2}\right) .
$$

For $p \geq 0$, we have

$$
\begin{aligned}
\omega_{p, 1} & =q^{2 p+1} \sum_{t=1}^{p} q^{-2 t} \\
\omega_{-p, 1} & =-q^{-2 p-1} \sum_{t=1}^{p} q^{2 t} \\
\omega_{p, 2} & =q^{\frac{5}{2}}\left(\sum_{t=1}^{p} q^{6(p-t)}+[2]_{q} \sum_{1 \leq s<t \leq p} q^{2(t-s)+6(p-t)}\right), \\
\omega_{-p, 2} & =q^{-\frac{5}{2}}\left(\sum_{t=1}^{p} q^{-6(p-t)}+[2]_{q^{-1}} \sum_{1 \leq s<t \leq p} q^{-2(t-s)-6(p-t)}\right) .
\end{aligned}
$$

Thus, for $p \in \mathbb{Z}$, we have

$$
\left.\omega_{p, 1}\right|_{q=-1}=-p,\left.\quad q^{-\frac{1}{2}} \omega_{p, 2}\right|_{q=-1}=p .
$$

Together with

$$
\left.\Phi_{1}\right|_{q=-1}=-2,\left.\quad \Phi_{3}\right|_{q=-1}=1,\left.\quad \Phi_{4}\right|_{q=-1}=2,\left.\quad \Phi_{5}\right|_{q=-1}=1,
$$


we have

$$
\begin{array}{r}
-\left.q^{-2} \omega_{i, 1} \omega_{j, 1} \omega_{k, 1} \Phi_{3} \Phi_{1}\right|_{q=-1}=-2 i j k, \\
\left.q^{-5-\frac{1}{2}} \omega_{i, 2} \omega_{j, 2} \omega_{k, 2} \Phi_{5} \Phi_{4} \Phi_{3} \Phi_{1}^{2}\right|_{q=-1}=8 i j k
\end{array}
$$

Thus we have

$$
\frac{J_{M_{i, j, k}}-1}{\Phi_{2}}=-2 i j k+8 i j k=6 i j k \quad\left(\bmod \Phi_{2}\right),
$$

which implies the assertion.

Acknowledgments. This work was partially supported by JSPS Research Fellowships for Young Scientists. The author is deeply grateful to Professor Kazuo Habiro, Professor Tomotada Ohtsuki, and Professor Toshie Takata for helpful advice and encouragement. In particular she is grateful to Professor Kazuo Habiro for many important discussions. She would like also to thank Professor Naoya Enomoto for discussions concerning the content of Section 4. Professor Paul Melvin for comments concerning the content of Remark 3.7. Professor Stefan Friedl for suggestions concerning the pictures in Figure 1.

\section{References}

[1] R. H. Bing, A homeomorphism between the 3-sphere and the sum of two solid horned spheres, Ann. of Math. (2) 56 (1952) 354-362.

[2] D. Cimasoni, Slicing Bing doubles. Algebr. Geom. Topol. 6 (2006), 2395-2415.

[3] J. C. Cha, Link concordance, homology cobordism, and Hirzebruch-type defects from iterated p-covers. J . Eur. Math. Soc. (JEMS) 12 (2010), no. 3, 555-610.

[4] J. C. Cha, T. Kim, Covering link calculus and iterated Bing doubles. Geom. Topol. 12 (2008), no. 4, 2173-2201.

[5] J. C. Cha, C. Livingston, D. Ruberman, Algebraic and Heegaard-Floer invariants of knots with slice Bing doubles. Math. Proc. Cambridge Philos. Soc. 144 (2008), no. $2,403-410$.

[6] T. D. Cochran, Derivatives of links: Milnor's concordance invariants and Massey's products. Mem. Amer. Math. Soc. 84 (1990), no. 427, x+73 pp.

[7] T. D. Cochran, S. Harvey, C. Leidy, Link concordance and generalized doubling operators. Algebr. Geom. Topol. 8 (2008), no. 3, 1593-1646.

[8] T. D. Cochran, P. Melvin, The Milnor degree of a 3-manifold. J. Topol. 3 (2010), no. $2,405-423$.

[9] T. D. Cochran, P. Melvin, Quantum cyclotomic orders of 3-manifolds. Topology 40 (2001), no. 1, 95-125. 
[10] M. Freedman, X. S. Lin, On the (A,B)-slice problem. Topology 28 (1989), no. 1, 91-110.

[11] S. Harvey, Homology cobordism invariants and the Cochran-Orr-Teichner filtration of the link concordance group. Geom. Topol. 12 (2008), no. 1, 387-430.

[12] K. Habiro, A unified Witten-Reshetikhin-Turaev invariants for integral homology spheres. Invent. Math. 171 (2008), no. 1, 1-81.

[13] K. Habiro, Claspers and finite type invariants of links. Geom. Topol. 4 (2000), 1-83.

[14] R. Kirby, P. Melvin, The 3-manifold invariants of Witten and Reshetikhin-Turaev for sl(2,C). Invent. Math. 105 (1991), no. 3, 473-545.

[15] H. R. Morton, P. Strickland, Jones polynomial invariants for knots and satellites. Math. Proc. Cambridge Philos. Soc. 109 (1991), no. 1, 83-103.

[16] T. Ohtsuki, A polynomial invariant of integral homology 3-spheres. Math. Proc. Cambridge Philos. Soc. 117 (1995), no. 1, 83-112.

[17] M. Petkovšek, H. S. Wilf, D. Zeilberger, A=B. With a foreword by Donald E. Knuth. With a separately available computer disk. A K Peters, Ltd., Wellesley, MA, 1996. xii+212 pp.

[18] N. Y. Reshetikhin, V. G. Turaev, Ribbon graphs and their invariants derived from quantum groups. Comm. Math. Phys. 127 (1990), no. 1, 1-26.

[19] N. Y. Reshetikhin, V. G. Turaev, Invariants of 3-manifolds via link polynomials and quantum groups. Invent. Math. 103 (1991), no. 3, 547-597.

[20] S. Suzuki, On the universal $s l_{2}$ invariant of ribbon bottom tangles. Algebr. Geom. Topol. 10 (2010), no. 2, 1027-1061.

[21] S. Suzuki, On the universal $s l_{2}$ invariant of boundary bottom tangles. Algebr. Geom. Topol. 12 (2012), 997-1057.

[22] S. Suzuki, On the universal $s l_{2}$ invariant of Brunnian bottom tangles, Math. Proc. Camb. Phil. Soc., 154 (2013), no.1, 127-143.

[23] S. Suzuki, On the colored Jones polynomials of ribbon links, boundary links, and Brunnian links. to appear in Banach Center Publ.

[24] T. Takata, On the set of the logarithm of the LMO invariant for integral homology 3-spheres. Math. Proc. Cambridge Philos. Soc. 145 (2008), no. 2, 349-361. 